\title{
Different Effects of Alcohol Exposure on Action and Outcome-Related Orbitofrontal Cortex Activity
}

\author{
Christian Cazares, ${ }^{1}$ Drew C. Schreiner, ${ }^{2}$ and Christina M. Gremel ${ }^{1,2}$
}

https://doi.org/10.1523/ENEURO.0052-21.2021

${ }^{1}$ The Neurosciences Graduate Program, University of California San Diego, La Jolla, CA 92093 and ${ }^{2}$ Department of Psychology, University of California San Diego, La Jolla, CA 92093

\begin{abstract}
Alcohol dependence can result in long-lasting deficits to decision-making and action control. Neurobiological investigations have identified orbitofrontal cortex (OFC) as important for outcome-related contributions to goal-directed actions during decision-making. Prior work has shown that alcohol dependence induces longlasting changes to OFC function that persist into protracted withdrawal and disrupts goal-directed control over actions. However, it is unclear whether these changes in function alter representation of action and outcome-related neural activity in OFC. Here, we used the well-validated chronic intermittent ethanol (CIE) exposure and withdrawal procedure to model alcohol dependence in mice and performed in vivo extracellular recordings during an instrumental task in which lever-press actions made for a food outcome. We found alcohol dependence disrupted goal-directed action control and increased OFC activity associated with leverpressing but decreased OFC activity during outcome-related epochs. The ability to decode outcome-related information, but not action information, from OFC activity following CIE exposure was reduced. Hence, chronic alcohol exposure induced a long-lasting disruption to OFC function such that activity associated with actions was enhanced, but OFC activity contributions to outcome-related information was diminished. This has important implications for hypotheses regarding compulsive and habitual phenotypes observed in addiction.
\end{abstract}

Key words: action; alcohol; compulsive; dependence; devaluation; orbitofrontal cortex

\section{Significance Statement}

Alcohol dependence produces long-lasting deficits in decision-making, including to actions made with regard to their outcome or consequence. This type of decision-making has been shown to involve orbitofrontal cortex (OFC). Here, using a mouse model of chronic alcohol exposure, we show that chronic alcohol exposure leads to bidirectional changes in activity of OFC neurons depending on the behavioral computation being performed. Mice that underwent previous chronic alcohol exposure showed increased OFC activity during actions for a food reward, but reduced OFC activity during reward-associated periods, and reduced OFC contribution to reward prediction.

\section{Introduction}

Alcohol use disorder (AUD) can result in decision-making deficits that can persist into protracted abstinence. In those suffering from AUDs, these deficits are thought to contribute to habitual and compulsive alcohol-seeking, a persistent vulnerability to relapse, and decrements in daily cognitive function (Stephens and Duka, 2008; Berre

\footnotetext{
Received February 8, 2021; accepted March 18, 2021; First published March 30, 2021.

The authors declare no competing financial interests.
}

et al., 2012; Reich and Goldman, 2015; Le Berre et al., 2017; Sebold et al., 2017; Bickel et al., 2018). With reports of alcohol dependence-induced functional and structural alterations across the cortex (Volkow et al., 1994, 1997; Laakso et al., 2002; Cardenas et al., 2011; Durazzo et al., 2011; Beck et al., 2012; Sjoerds et al., 2013; Thayer et al.,

\footnotetext{
Author contributions: C.C. and C.M.G. designed research; C.C. performed research; C.C., D.C.S., and C.M.G. contributed unpublished reagents/analytic tools; C.C. and D.C.S. analyzed data; C.C., D.C.S., and C.M.G. wrote the paper.
} 
2016), it is highly likely that a broad array of computations normally contributing to efficacious decision-making are also altered. Identifying which computations are disrupted along with any corresponding aberrant activity patterns would offer a starting point for mechanistic investigations into cortical circuit alterations that produce these longlasting impairments in decision-making.

One such cortical circuit that often shows long-lasting dependence-induced disruptions is the orbitofrontal cortex (OFC). Abstinent AUD patient studies generally report a hypoactive OFC both at baseline and during adaptive decision-making (Volkow et al., 1994, 1997; Boettiger et al., 2007; Sjoerds et al., 2013; Reiter et al., 2016) but also report OFC hyperactivity to stimuli and related approach behaviors (Wrase et al., 2002; Hermann et al., 2006; Reinhard et al., 2015), reminiscent of OFC hyperactivity reported in patients with other psychiatric conditions, including obsessive compulsive disorder (Milad and Rauch, 2012; Pauls et al., 2014; Robbins et al., 2019; Lüscher et al., 2020). This dichotomy of effects suggests that longlasting perturbations to OFC circuitry induced by alcohol dependence may differentially alter the computations performed by OFC neurons in response to information coming into OFC. Hence, initial investigations into computations performed by OFC during decision-making and their longlasting disruption in alcohol dependence would provide a framework with which to investigate broader circuit mechanisms contributing to observed OFC dysfunction.

Several lines of evidence implicate the OFC as a key contributor to computations that can contribute to valuebased decision-making processes (Fellows, 2007; Wallis, 2007; Gremel and Costa, 2013; Stalnaker et al., 2015; Padoa-Schioppa and Conen, 2017) as well as to compulsive control (Milad and Rauch, 2012; Pauls et al., 2014; Robbins et al., 2019; Lüscher et al., 2020). OFC neurons will modulate firing rate when subjects make a lever press (Gremel and Costa, 2013) and when presented with an appetitive outcome (Rolls et al., 1996; Wallis, 2011). Functional manipulations to OFC activity have generally supported a role for OFC in using outcome-related information to control decision-making (Gremel and Costa, 2013; Rhodes and Murray, 2013; Baltz et al., 2018; Malvaez et al., 2019). Interestingly, increased OFC activity has also been functionally implicated in contributing to compulsive control over behavior (Ahmari et al., 2013; Burguière et al., 2013; Pascoli et al., 2015, 2018), with previous work showing that increased OFC activity and

This work was supported by National Institute on Alcohol Abuse and Alcoholism Grants R00AA021780 and R01AA026077 (to C.M.G.) and F31AA027439 (to D.C.S.), Whitehall and Brain and Behavior Foundations (C.M. G.), and the National Science Foundation GRFP Grant DGE-1650112 (to C.C.).

Acknowledgements: We thank undergraduate research assistant Mariela Lopez Valencia for technical assistance and Roy Jungay for animal care assistance.

Correspondence should be addressed to Christina M. Gremel at cgremel@ucsd.edu.

https://doi.org/10.1523/ENEURO.0052-21.2021

Copyright (C) 2021 Cazares et al.

This is an open-access article distributed under the terms of the Creative Commons Attribution 4.0 International license, which permits unrestricted use, distribution and reproduction in any medium provided that the original work is properly attributed. downstream output supports compulsive lever pressing for dopamine neuron stimulation (Pascoli et al., 2018).

Likewise, animal models of alcohol dependence have revealed long-lasting dependence-induced disruptions to OFC-dependent processes, including behavioral flexibility and outcome devaluation (Badanich et al., 2011; Kroener et al., 2012; Lopez et al., 2014; Fernandez et al., 2017; Renteria et al., 2018, 2020). Chronic heavy alcohol consumption in non-human primates, as well as prior chronic alcohol exposure in mice, results in long-lasting changes to OFC intrinsic excitability, synaptic transmission, and increases in dendritic spine density of OFC neurons (Badanich et al., 2013; McGuier et al., 2015; Nimitvilai et al., 2016, 2017; Renteria et al., 2018). Similar to findings in chronically drinking non-human primates (Nimitvilai et al., 2017), previous work has reported that chronic intermittent ethanol (CIE) vapor exposure and withdrawal procedures in mice led to a reduction in OFC excitability (Renteria et al., 2018). ClE-exposed mice also showed an insensitivity to outcome devaluation in protracted withdrawal, characterized as a reduction in goal-directed control and an increased reliance on habitual control over lever pressing for food. Notably, artificially increasing the activity of OFC projection neurons was sufficient to restore sensitivity to outcome devaluation in CIE-exposed mice (Renteria et al., 2018). To this end, the observed dependence-induced deficits in decision-making are hypothesized to include alterations to OFC activity critical for decision-making processes. However, whether this includes alterations to OFC activity during actions and outcome-related epochs is unknown.

Here, we examined CIE exposure-induced disruptions to OFC activity during protracted withdrawal in an instrumental task where actions are made for a food outcome. We used an adapted action contingency task, historically termed action differentiation (Platt et al., 1973; Kuch, 1974; Yin, 2009; Fan et al., 2012). In this task, mice must learn to press and hold a lever down beyond a fixed minimum duration to earn a food reward. The structure of the task allows us to look at OFC activity at the onset, during, and offset of lever presses, as well as during outcomerelated epochs. Prior works have found that alcoholexposed rats and mice show similar acquisition of lever-press performance compared with naive controls, but outcome devaluation and contingency degradation procedures (Corbit et al., 2012; Lopez et al., 2014; Morisot et al., 2019; Renteria et al., 2018; Barker et al., 2020) have shown that such lever pressing is controlled by habitual, instead of goal-directed, processes. Thus, in a subset of mice, we performed outcome devaluation testing procedures after acquisition. Replicating previous findings, we show that air and CIE mice acquire similar lever pressing performance. However, subsequent outcome devaluation testing showed that such lever pressing was under habitual control in CIE mice and goal-directed control in air mice. When we examined OFC activity during behavioral acquisition, we found that prior induction of alcohol dependence led to higher OFC firing rates related to lever-pressing, but reduced firing rates during periods associated with outcome delivery. Decoder analyses on 
OFC activity showed reduced accuracy to classify outcome related information in $\mathrm{CIE}$-exposed mice compared with controls. Thus, while CIE led to increased activity related to actions, it reduced OFCs normal representation of outcome-related information that may be important for goal-directed decision-making.

\section{Materials and Methods}

\section{Animals}

Male and female C57BL/6J mice $(n=15,9$ males, 6 females or non-recording experiments; $n=18,17$ males, 1 female for recording experiments) were housed two to five per cage under a 14/10 h light/dark cycle with access to food (Labdiet 5015) and water ad libitum unless stated otherwise. C57BL/6J (The Jackson Laboratory) mice were at least six weeks of age before intracranial micro-array implant and at least $52 \mathrm{~d}$ of age before vapor procedures or behavioral training. Investigators were not blind to the experimental groups. The Animal Care and Use Committee of the University of California, San Diego approved all experiments and experiments were conducted according to the $\mathrm{NIH}$ guidelines.

\section{Surgical procedures}

Animals under isoflurane anesthesia were implanted with a stereotaxically guided fixed micro-array consisting of four-rows of four platinum-plated tungsten electrodes (35- $\mu \mathrm{m}$ tip, Innovative Neurophysiology), with electrodes spaced $150 \mu \mathrm{m}$ apart, and rows $150 \mu \mathrm{m}$ apart. The dearth of female mice in the recording study was because of problems with female mice not being able to maintain and carry the electrode implant through CIE procedures and behavioral testing. To maximize targeting of the OFC, arrays were centered at the following coordinates from bregma: A, $2.5 \mathrm{~mm}$; M/L, $1.3 \mathrm{~mm}$;, $2.0 \mathrm{~mm}$. An additional bilateral craniotomy was made over the posterior cerebellum for placement of screws wrapped with the electrical reference wire attached to the micro-array. After testing, mice were euthanized, and brains extracted and fixed in $4 \%$ paraformaldehyde. Micro-array placement was qualified by examining tracts in $50-$ to $100-\mu \mathrm{m}$-thick brain slices under a macro fluorescence microscope (Olympus MVX10). A subset of micro-arrays was dyed

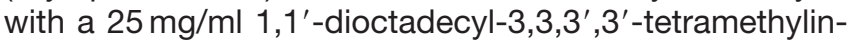
docarbocyanine perchlorate (Dil) solution in 200 proof ethanol (Sigma) for placement verification. All surgical and behavioral experiments were performed during the light portion of the cycle.

\section{CIE exposure and repeated withdrawal}

One to two weeks after micro-array implant surgeries for recording mice, all mice were exposed to four rounds of ethanol vapor or air (Becker and Hale, 1993; Becker and Lopez, 2004; Lopez and Becker, 2005; Griffin et al., 2009; Renteria et al., 2018). Each round consisted of $16 \mathrm{~h}$ of vapor exposure followed by an 8-h withdrawal period, repeated for four consecutive days. The CIE procedure is designed to repeatedly induce alcohol withdrawal syndrome after long periods of alcohol exposure, a key criterion in the diagnosis of alcohol dependence (Lopez and Becker, 2005). Ethanol was volatilized by bubbling air through a flask containing $95 \%$ ethanol at a rate of 2-3 I/ min. The resulting ethanol vapor was combined with a separate air stream to give a total flow rate of $\sim 10 \mathrm{l} / \mathrm{min}$, which was delivered to the mice housed in Plexiglas chambers (Plas Labs Inc). Mice were not pretreated with a loading dose of ethanol or pyrazole to avoid confounding effects of stress that can bias reliance on habitual control, as well as to avoid the effects of pyrazole on neural activity, including actions at the NMDA receptor (Pereira et al., 1992; Becker and Lopez, 2004; Dias-Ferreira et al., 2009). Blood ethanol concentrations (BECs) were collected at the end of each round from separate, non-experimental mice to avoid previously reported stress effects on decision-making from blood extraction on air mice (mean \pm SEM BEC $=29.53 \pm 2.36 \mathrm{~mm}$ ). BEC assays that experienced technical errors were excluded from this measurement.

\section{Behavioral task}

We adapted a lever press hold down task previously used to assay the timing of decision-making actions in mice (Yin, 2009; Fan et al., 2012). Mice were trained in standard operant chambers with one lever extended to the left (or right) of a food magazine and a house light on the opposite wall within sound-attenuating boxes (MedAssociates). Two days before training, mice were food restricted and maintained at $85-90 \%$ of their baseline body weight throughout training and testing.

\section{Magazine training}

On the first day, mice were trained to retrieve pellets from the food magazine (no levers present) on a random time (RT) schedule, with a pellet outcome delivered on average every $120 \mathrm{~s}$ for $60 \mathrm{~min}$.

\section{Continuous reinforcement}

The next $3 d$ the left (or right) lever was present the entire duration of the session. Lever presses were rewarded on a continuous reinforcement (CRF) schedule for up to 15 (CRF day 1), 30 (CRF day 2), or 60 (CRF day 3) pellet deliveries or until 60-90 min had passed. For electrodeimplanted animals, an additional CRF training day ( $4 \mathrm{~d}$ total) was administered with the implant connected to the amplifier board to habituate the animal to the tethered connection.

\section{Lever press hold down training}

The action differentiation task required lever press durations to exceed a duration criterion assigned before the start of the daily session. This criterion was the minimum duration of time the animal was required to hold the lever in a depressed position to receive a reward. Each session began with the house light turning on and the left (or right) lever being extended for the duration of the session. Lever pressing was self-initiated and self-paced without an imposed trial structure (i.e., the lever was never retracted until the session was complete). Reward delivery occurred at the offset of the lever press only if the hold down timer exceeded the session's assigned duration criterion. 
Sessions were completed when 30 outcomes (non-recording animals) or 60 outcomes (recording animals) were earned or after $90 \mathrm{~min}$, whichever came first. The lever press duration criterion for the first $5 \mathrm{~d}$ was $800 \mathrm{~ms}$, followed by $5 \mathrm{~d}$ ( $4 \mathrm{~d}$ in three animals because of loss of head-cap implant) of a lever press duration requirement of $1600 \mathrm{~ms}$.

\section{Devaluation testing}

Following the last day of hold down testing in the behavioral cohort, mice were habituated to a novel cage and $20 \%$ sucrose solution for $1 \mathrm{~h}$ each. Devaluation testing through sensory-specific satiation was conducted across $2 \mathrm{~d}$ and consisted of a valued day and a devalued day. For the valued day, the mice were allowed to prefeed for $1 \mathrm{~h}$ on $20 \%$ sucrose solution. For the devalued day, mice could prefeed for $1 \mathrm{~h}$ on the pellet outcome previously earned in the lever press hold down task. Mice that did not consume enough pellets $(<0.1 \mathrm{~g})$ or sucrose $(<0.1 \mathrm{ml})$ during prefeeding were excluded from subsequent analysis (CIE cohort, $n=1$ ). Each day immediately following prefeeding, mice were placed into their respective operant chamber for $10 \mathrm{~min}$, where the number and duration of lever presses made were recorded, but no outcome was delivered. Investigators were not blind to the experimental groups. Valued and devalued days were counterbalanced and run across consecutive days. Response rate comparisons between valued and devaluated days were made by normalizing each mouse's test day response rate to the average response rate of their corresponding last $2 \mathrm{~d}$ of 1600 -ms duration criterion sessions using the following formula:

$$
R R_{\text {Test Day } \div} \overline{R R_{1600-4}+R R_{1600-5}}
$$

\section{Electrophysiological recordings and spike sorting}

Spike activity and local field potentials were recorded using an RHD2000 USB interface board system connected to an amplifier board via a serial peripheral interface (SPI) cable (Intan Technologies). Electrode signals were amplified, digitized at $30 \mathrm{kHz}$ and filtered between $0.1 \mathrm{~Hz}$ and $6 \mathrm{kHz}$ for spikes and 0.1 and $600 \mathrm{~Hz}$ for local field potentials. Initial sorting occurred before each testing session using an online-sorting algorithm (OpenEphys; Siegle et al., 2017). Behavior events that occurred inside the operant boxes were timestamped in synchronization in OpenEphys with neural activity using transistor-transistor logic (TTL) pulses collected at a 10-ms resolution from Med Associates SuperPort Output cards. Spike data were resorted offline (Offline Sorter, Plexon) using a T-Distribution Expectation-Maximization Scan algorithm in 3D feature space (Shoham et al., 2003). This allowed for the identification of neuronal activity units based on waveform, amplitude, and inter spike interval histogram (no spikes during a refractory period of $1.4 \mathrm{~ms}$ ). After sorting, each isolated cluster of waveforms was then manually inspected, and biologically implausible waveform clusters were removed from further analysis. To ensure high signal-to-noise quality of each waveform cluster, waveforms 2 standard deviations (SDs) greater than the clustered population mean were excluded from the analyses. Units with $<1000$ spike waveforms captured within an entire recording session or that did not show consistent activity across a recording session were not included in our analyses. Before each recording session, mice were exposed to a brief (10-20 s) bout of low-dose isoflurane anesthesia to connect the implant with the recording cable. To avoid confounding effects of anesthesia on brain activity, mice were then moved into the procedure room and monitored for a minimum of 30 min before placing them in the operant chamber and initiating the session.

\section{Identification of significantly modulated units}

To initially examine task-related neural activity, for each previously isolated recorded unit we constructed a perievent histogram (PETH) around time-stamped lever-press and reward delivery events, such that neural activity was binned into 20-ms bins and averaged across events to analyze amplitude and latency during the recorded behaviors. Per-unit PETHs were then smoothed using a Gaussianweighted moving average over three bins $(60 \mathrm{~ms})$. Using the distribution of the PETH from 10,000 to 2000 ms before lever press onset as baseline activity, we focused our analysis on a period $2000 \mathrm{~ms}$ before to $10,000 \mathrm{~ms}$ after task-related events. A task-related neuron was up-modulated if it had a significant increase in firing rate defined as at least four bins $(80 \mathrm{~ms})$ with a firing rate larger than a threshold of $95 \%$ confidence interval above baseline activity during the period from 2000 before to $3000 \mathrm{~ms}$ after each task event. A task-related neuron was down-modulated if it had a significant decrease in firing rate if at least four consecutive bins $(80 \mathrm{~ms})$ had a firing rate smaller than a threshold of $95 \%$ confidence interval below baseline activity during the period from 2000 before to $3000 \mathrm{~ms}$ after each task event (Jin and Costa, 2010). The onset of significantly modulated task-related activity was defined as the first of these four-consecutive significant PETH bins. To examine the net effect of CIE on OFC activity as animals performed the task, we combined these up-modulated and down-modulated unit populations for subsequent population analyses.

\section{Population analyses \\ Performance-related spike activity}

To investigate differences in peri-event spike activity between lever presses that were rewarded or not, spike timestamps occurring $10,000 \mathrm{~ms}$ before to $10,000 \mathrm{~ms}$ after individual lever press events were split into successes (lever press duration exceeded session's criterion duration) and failures (lever press duration did not exceed session's criterion duration). Performance segmented neural activity was then binned into 20 -ms bins, averaged across events, and then smoothed using a Gaussianweighted moving average over three bins (60 ms), resulting in two PETHs per unit (successes or failures). Individual PETHs were then converted to $z$-scores using the mean and $S D$ of the firing rate during a baseline period occurring 10,000-2000 before lever press onset. Per-unit $z$-scored PETHs were then averaged by treatment group to construct population response profiles for each group. 
Population spike activity from the last the two sessions of 1600 -ms duration criteria was grouped such that a minimum of one session per animal was included. Population spike activity traces were then smoothed with MATLAB's Savitzky-Golay smoothdata method using a 400-sample sliding window for visual display purposes only.

\section{Ongoing lever press-related spike activity}

To investigate differences in spike activity during ongoing lever-presses, each lever press duration was first calculated by subtracting the lever press onset timestamp from lever press offset timestamp. Each lever press duration was then segmented into four equivalent segment bins (i.e., $0-25 \%, 25-50 \%, 50-75 \%, 75-100 \%$ of lever press duration), and all spikes occurring within each of these duration bins were counted and calculated as a proportion of all spikes that occurred during that entire lever press.

To investigate differences in firing rate changes between different lever press durations, lever presses were first grouped into four quartiles determined by the distribution of lever press durations within each individual recording session. Quartile-grouped spike activity occurring $10,000 \mathrm{~ms}$ before to $10,000 \mathrm{~ms}$ after lever press onset was then binned into 20-ms bins, averaged across lever presses, and then smoothed using a Gaussian-weighted moving average over three bins $(60 \mathrm{~ms})$, resulting in four PETHs per unit, one for each duration quartile. To account for variable lever press durations, PETHs were converted to z-scores using the mean and SD of activity occurring before the onset of the lever press proportionate in duration to the average lever press duration within each quartile. Individual lever press activity from these PETHs were then segmented into four equivalent segment bins (i.e., $0-$ $25 \%, 25-50 \%, 50-75 \%, 75-100 \%$ of lever press duration). Per-unit, baseline z-scored traces were then averaged across the four duration segments within quartile and treatment groups to construct population response profiles.

\section{Neural decoding of task performance from spike activity}

For all units from recording sessions in which a minimum of 10 lever presses exceeded the session's lever press duration criterion, spike timestamps occurring $2000 \mathrm{~ms}$ before to $10,000 \mathrm{~ms}$ after individual lever press events were binned into 1-ms bins and labeled by lever press outcome (success or failure to exceed the session's lever press duration criterion). These peri-event rasters were then segmented by treatment groups and task event (lever press onset or offset) and used to train a model to classify successful lever presses. The classifier, a support vector machine model implemented in MATLAB with the NDT toolbox, was trained and tested at $100-\mathrm{ms}$ steps with a bin width of $200 \mathrm{~ms}$ (Meyers, 2013). For each of these time points, the classifier used 10 cross-validation splits to segment per-unit firing rates from randomly selected lever press events into training $(90 \%)$ and testing $(10 \%)$ sets for 500 resampling runs. Significance at each of these timepoints was tested by first creating 5 null distributions of decoding accuracy with 500 resampling runs each in which the performance labels were shuffled. The accuracy of our decoder was then compared with these null distributions across all time points.

\section{Statistical procedures}

Statistical significance was defined as an $\alpha$ of $p<0.05$. Statistical analysis was performed using GraphPad Prism 8.3.0 (GraphPad Software) and custom MATLAB R2019a (MathWorks) scripts using a PC desktop with Windows 10. Acquisition data, including lever presses, response rate, and proportion of lever presses that were rewarded were analyzed using two-way repeated measures ANOVA (session $\times$ treatment) unless otherwise noted. For outcome devaluation testing, two-way repeated measure ANOVAs (value $\times$ treatment) with preplanned post hoc Sidak's multiple comparison testing were performed to examine whether outcome devaluation reduced lever pressing on the devalued compared with valued day within each group. For peri-event spike activity comparisons, per-unit average z-scored firing rates were binned into four 250-ms bins before the lever press onset, or after lever press offset and after reward delivery, respectively. Within treatment groups, we performed two-way repeated measure ANOVAs (bin $\times$ outcome) to examine differences in spike activity between lever presses that failed or succeeded to exceed the session's lever press duration criteria, with post hoc Sidak's multiple comparison testing to determine bins in which differences were pronounced. Two-way repeated measure ANOVAs (bin $x$ treatment) were performed to examine differences in spike activity between treatment groups, with post hoc Sidak's multiple comparison testing to determine bins in which differences were pronounced. Two-way repeated measure ANOVAs (segment $\times$ treatment) were performed to examine group differences in the proportions of spikes occurring between lever press duration segments. Between group comparisons of decoder accuracy were made with twoway repeated measure ANOVAs (bin $\times$ treatment), with post hoc Benjamini and Hochberg multiple comparison testing to examine in which of the 100-ms bins were differences pronounced. When appropriate, mixed-effect analyses were conducted in lieu of repeated measures ANOVAs (e.g., when data points were missing because of loss of implant). Data are presented as mean \pm SEM.

\section{Code accessibility}

The code/software described in the paper is freely available online at https://github.com/gremellab/CIEOFCHOLD. The code is available as Extended Data 1..m files). All data generated or analyzed during this study are available at $\mathrm{https} / / / \mathrm{doi}$. org/10.6084/m9.figshare.12605225.v1.

\section{Results}

\section{Induction of ethanol dependence disrupts goal- directed control over lever pressing}

We employed a well-validated model of CIE vapor exposure and repeated withdrawal (Becker and Hale, 1993; Becker and Lopez, 2004; Lopez and Becker, 2005; Griffin 


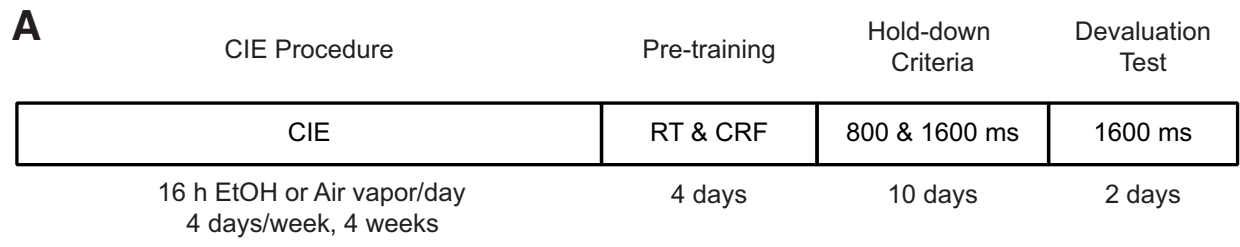

C
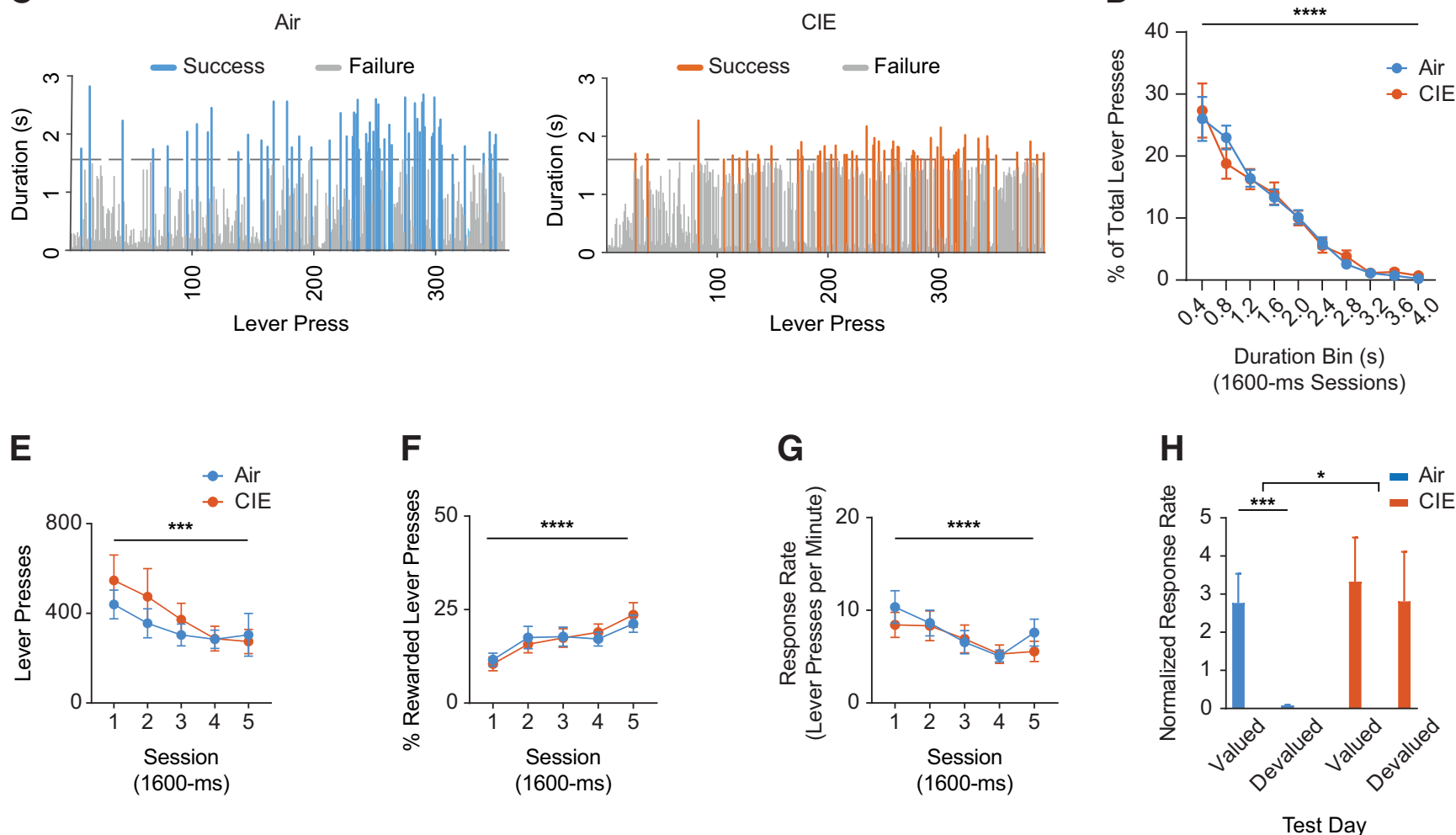

Figure 1. Effects of alcohol dependence on lever press acquisition $(\boldsymbol{A}-\mathbf{G})$ and outcome devaluation $(\boldsymbol{H})$. $\boldsymbol{A}$, Experimental timeline starting with the CIE procedure, subsequent RT delivery of outcome, fixed-ratio CRF on lever press, five daily sessions of 800-ms, and then five daily sessions of 1600-ms, lever press duration criterion sessions and, lastly, two subsequent days of outcome devaluation (DV) testing. B, Schematic of lever press duration performance. Lever presses exceeding the session's minimum hold-down duration criterion were rewarded only at the offset of the lever press. $\boldsymbol{C}$, Example lever press performance from individual air (left) and CIE (right) mice during a 1600-ms hold-down duration criterion session late in training. Distribution of lever press durations (D), average total lever presses $(\boldsymbol{E})$, average percentage of rewarded lever presses $(\boldsymbol{F})$, and average response rate through 1600 -ms lever press duration criterion sessions $(\boldsymbol{G})$. $\boldsymbol{H}$, Average normalized response rate in valued and devalued states throughout devaluation testing. Lever press duration distributions throughout acquisition and predevaluation test food consumption are shown in Extended Data Figure 1-1. Data points represent mean \pm SEM; ${ }^{\star} p<0.05,{ }^{\star \star \star} p<0.001,{ }^{\star \star \star \star} p<0.0001$

et al., 2009). Mice were exposed to periods of ethanol (CIE) or air (air) vapor and subsequent withdrawal over a period of four weeks (six vapor cohorts; air: $n=17$, CIE: $n$ $=16$; Fig. 1A). CIE procedures produced mean BECs in ethanol-exposed mice in line with previous reports $(29.53 \pm 2.36 \mathrm{~mm}$; Lopez and Becker, 2005; Renteria et al., 2018). Alcohol withdrawal has been delineated into two phases; an immediate acute withdrawal period (2$3 \mathrm{~d}$ ), followed by a protracted period extending at least three months (Heilig et al., 2010). To examine OFC activity and related behavior during this protracted withdrawal period, food-restricted mice began instrumental training and testing procedures $5 \mathrm{~d}$ after their last vapor exposure.
To examine the effects of prior CIE procedures on OFC activity during action and outcome-related epochs in decision-making, we adapted an instrumental task examining action differentiation (Fig. 1A), where a mouse must learn to press and hold a lever down beyond a fixed minimum duration to earn a reward (Yin, 2009; Fan et al., 2012). Throughout training, mice learned to press and hold down a lever beyond a predetermined minimum duration to earn a food pellet on release of the lever. Mice self-initiated and self-terminated every lever press in the absence of any extrinsic cues signaling lever press duration. Importantly, reward delivery occurred only at the offset of a lever press that exceeded the duration criteria (Fig. $1 B, C$ ), preventing the use of reward presence to 
signal lever press termination. Thus, this task produces discretized behavior epochs conducive to neural activity analysis (e.g., lever-press onset, during the lever-press, lever-press offset, and outcome delivery).

Following vapor procedures, air and CIE mice underwent action differentiation training, with the initial criterion for lever press duration set at 800 -ms for five daily sessions, followed by five daily sessions of a 1600 -ms criterion (see Materials and Methods). Representative sessions from one air and one CIE mouse on a 1600-ms criteria day are shown in Figure $1 C$, suggesting that mice show a distribution of lever press durations that approximate the duration criterion. This distribution of lever presses was similar between air and CIE mice (1600-ms criteria training sessions, two-way repeated measures ANOVA (bin $\times$ treatment); no interaction; main effect of bin: $F_{(9,252)}=53.56$, $p<0.0001$ ), and importantly, each group showed evidence of learning with rightward shifts in their corresponding lever press duration distributions following the switch from 800 - to 1600-ms training criteria (Extended Data Fig. 1-1A,B). We focused our examinations on behavior throughout the 1600-ms criterion sessions, after mice had learned the action differentiation rule and had shifted to a longer duration contingency. Air and CIE mice showed similar levels of lever pressing that increased across sessions (mixed-effects repeated measures ANOVA (session $\times$ treatment); no interaction; main effect of session only: $F_{(4,121)}=5.36, p<0.0001$; Fig. $1 E$ ). This task was similarly challenging for air and $\mathrm{CIE}$ mice, and only $\sim 25 \%$ of total lever presses in air and CIE mice exceeded the duration criterion within each session (mixed-effects repeated measures ANOVA (session $\times$ treatment); no interaction; main effect of session: $F_{(4,121)}=12.28, p<0.0001$; Fig. $1 F$ ). Further, CIE treatment did not alter response rates, which increased across sessions for both air and CIE mice (mixed-effects repeated measures ANOVA (session $\times$ treatment); no interaction; main effect of session: $F_{(4,121)}=12.28$, $p<0.0001)$. Thus, as expected, CIE and air mice show similar lever press acquisition for food reward.

Examination of lever press performance as discussed above, however, cannot distinguish whether lever press is under goal-directed of habitual control. To examine whether the observed lever pressing was under different action controllers in CIE compared with air mice, we performed outcome devaluation procedures and testing following training on the $1600-m s$ duration criterion. In outcome devaluation testing, a reduction in response rates following prefeeding with the outcome normally earned through lever pressing has been defined as a measure of goal-directed control (Dickinson, 1985). Prior work has found that alcohol dependence reduces the contribution of goal-directed control to lever pressing for food (Dickinson, 1985; Lopez et al., 2014; Renteria et al., 2018) within the time frame examined in the present experiment (Renteria et al., 2018). We subjected a subset of air and CIE mice to sensory-specific satiation of food pellets previously earned by lever pressing or to a previously habituated control outcome (20\% sucrose solution). In each of the two consecutive test days, mice had ad libitum access to either pellets (devalued state) or sucrose solution (valued state) for $1 \mathrm{~h}$ before measuring non-reinforced lever press responses in the operant chamber throughout each 10-min 1600-ms duration criterion session. While air mice clearly reduced response rates (normalized to the response rate during last two days of acquisition) in the devalued state compared with valued state, CIE mice did not. A two-way repeated measures ANOVA (value $\times$ treatment) revealed different patterns of responding in treatment groups (interaction: $F_{(1,22)}=6.961, p=0.015$; main effect of value only: $\left.F_{(1,22)}=12.54, p=0.001\right)$. Post hoc analysis on normalized response rates revealed a difference between valued and devalued test sessions in air mice $(p=0.0002)$, but not in CIE mice $(p>0.5)$. As air and $\mathrm{CIE}$ mice consumed similar amounts of both outcomes during the prefeeding periods (Extended Data Fig. 1-1C), the data show that lever press performance in CIE mice was not controlled by goal-directed processes.

\section{OFC populations differentially encode lever-pressing and outcome-related components}

Given that the deficits in outcome devaluation observed in CIE mice have been shown to involve OFC (Gourley et al., 2013; Gremel and Costa, 2013; Rhodes and Murray, 2013; Gremel et al., 2016; Renteria et al., 2018), and OFC modulates activity during outcome-related epochs (Rolls et al., 1996; Wallis, 2011), we hypothesized that alcohol exposure would disrupt OFC neural activity related to lever press and outcome-related epochs in our task. We examined OFC activity in relation to task epochs in a subset of the mice that had been implanted with chronic indwelling micro-electrode arrays into the OFC before the start of CIE procedures (five vapor cohorts; air $n=9$, CIE $n=9$; Fig. $2 A$ ).

We focused on OFC activity data collected during the last two sessions of the 1600-ms duration criterion, a time point during which animals from both groups most proficiently performed the task. Putative single OFC unit spike activity was aligned to timestamps collected each time a lever press onset, offset, or pellet reward delivery occurred (see Materials and Methods). Importantly, there was no effect of $\mathrm{CIE}$ exposure on average baseline firing rates $(p>0.05$; Fig. $2 B$ ). More OFC units in CIE mice $(67 \%)$ than air mice $(54 \%)$ showed significantly altered firing rates during any task-related epochs (Fig. 2C; $\chi_{1,667}^{2}=10.21, p<0.002$; see Materials and Methods). However, in both groups, we found similar proportions of OFC units that significantly up-modulated (increased firing rate) or down-modulated (decreased firing rate) across lever press onset, lever press offset, and outcome delivery epochs $\left(\chi^{2} s<3.76, p s>0.05\right.$; Fig. $\left.2 D\right)$. Furthermore, we found that in both air and CIE mice, individual OFC units usually altered activity across multiple epochs (e.g., both the onset and offset of a lever press; Fig. $2 E ; \chi^{2}{ }_{6,394}=$ $8.04, p<0.24)$, with relative high percentages of OFC units encoding action onset, action offset, and reward (air = 18\%; CIE =23\%). Air and CIE mice showed similar numbers of significantly modulated units/mouse across events (lever press onset (air $=7.83 \pm 1.8, \mathrm{CIE}=7.3 \pm 1.8$ ); lever press offset (air $=8.06 \pm 1.6, \mathrm{CIE}=7.11 \pm 1.7$ ), reinforcement delivery (air =6.11 $\pm 2.3, \quad \mathrm{CIE}=4.61 \pm 1.33$ ). Altogether, this suggests that the OFC populations 
A

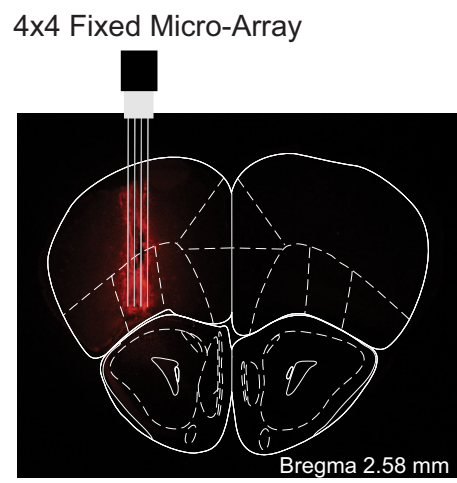

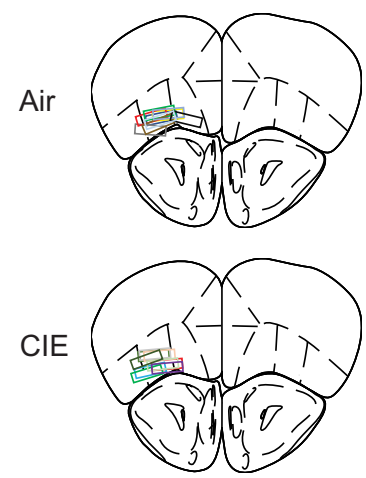

B

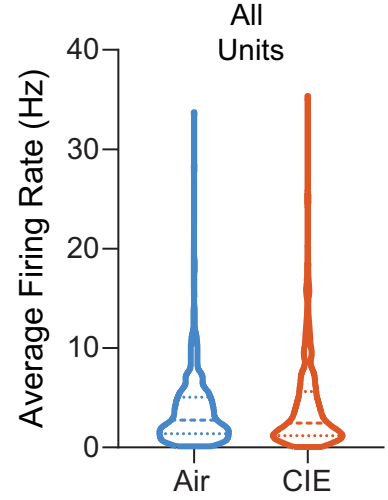

C

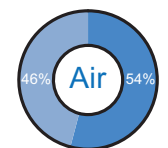

Total Units $=401$

- Significant

- Not Significant

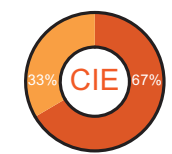

Total Units $=266$

- Significant

- Not Significant
D

Air CIE

Up-Modulated

B Down-Modulated
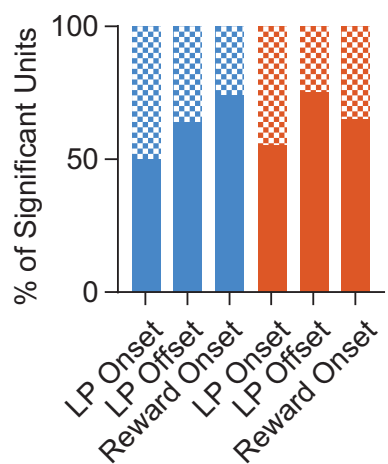

$\mathbf{E}$ Approximate Percentage of Total Significant Units Across Task Components

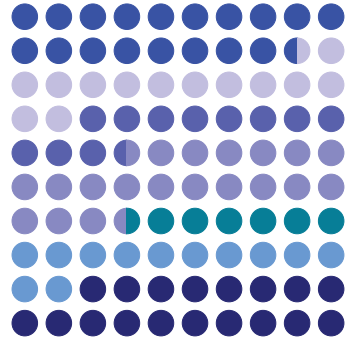

Total Significant

Units $=217$
CIE

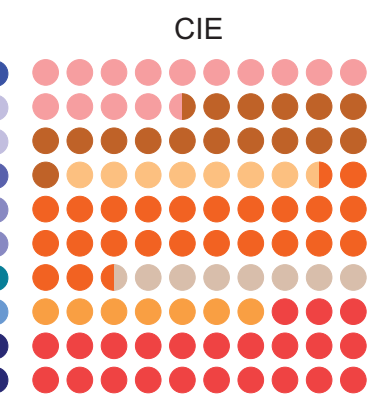

Total Significant

Units $=177$

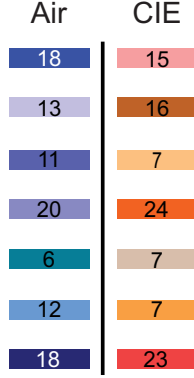

23

LP Onset

LP Offset

Reward Onset

LP Onset + LP Offset

LP Onset + Reward Onset

LP Offset + Reward Onset

LP Onset + LP Offset +

Reward Onset

Figure 2. $\boldsymbol{A}-\boldsymbol{E}$, OFC activity correlates of lever pressing and outcome delivery. $\boldsymbol{A}$, Representative image of fixed micro-array implant over the orbitofrontal cortex. Implant locations segmented by air and CIE groups. A subset of micro-arrays was dyed with a $25 \mathrm{mg} /$ $\mathrm{ml}$ Dil solution in 200 proof ethanol for placement verification. $\boldsymbol{B}$, Average firing rates from all captured units during a baseline period ( -5 to $-2 \mathrm{~s}$ before lever press onset) for air and CIE cohorts. $\boldsymbol{C}$, Proportion of all captured units in which firing rates significantly deviated from a baseline period ( -5 to $-2 \mathrm{~s}$ before lever press onset) for air ( $\sim 54 \%)$ and CIE ( $66 \%)$ groups. $\boldsymbol{D}$, Percentage of units that significantly increased or decreased their firing rates from baseline in relation to lever press onset, offset, and food pellet reward delivery. $\boldsymbol{E}$, Percentage of units that significantly changed their firing rates from baseline in relation to discrete task events (lever press onset: air: $\sim 18 \%$, CIE: $\sim 15 \%$; lever press offset: air: $\sim 13 \%$, CIE: $\sim 16 \%$; reward delivery: air: $\sim 11 \%$, CIE: $\sim 7 \%$ ) as well as multiple task components (lever press onset and offset: air: $\sim 20 \%$, CIE: $\sim 24 \%$; lever press onset and reward delivery: air: $\sim 6 \%$, CIE: $\sim 7 \%$; lever press offset and reward delivery: air: $\sim 12 \%$, CIE: $\sim 7 \%$; lever press onset, offset, and reward delivery: $\sim 18 \%$, CIE: $\sim 23 \%$ ).

normally recruited during this instrumental task were largely not altered following the induction of alcohol dependence.

\section{Prior CIE procedures enhances OFC lever press- related activity}

While we observed similar recruitment of OFC populations during behavior, it may be that the magnitude and patterns of OFC activity during task-related epochs are different between the two groups. We first asked whether CIE would alter OFC activity associated with the initiation of lever pressing. We examined the firing rate activity of all significantly modulated units during the 1000-ms period preceding lever press onset, as shown in the normalized activity peri-event heatmaps in both air and CIE animals (Fig. 3A). We found greater increases in baseline normalized OFC firing rates before the onset of lever pressing in CIE mice compared with air mice (Fig. 3C). A two-way repeated measures ANOVA (250-ms bin $\times$ treatment) showed no interaction, but a main effect of treatment only $\left(F_{(1,1032)}=17.39, p<0.0001\right)$. This increase in firing rates was also present when we examined upmodulated and down-modulated CIE populations separately (Extended Data Fig. 3-1A,B), suggesting an overall increase in action-related OFC activity in CIE mice.

We next asked whether activity associated with lever press initiation reflected future performance outcomes, i. e., were firing rates different for lever presses that were eventually rewarded? To this end, we grouped lever press durations by whether they successfully exceeded the lever press duration criterion or not (Fig. 3B). We did not find evidence of predictive coding of successful performance in either air or CIE mice (Extended Data Fig. 3-1C,D). 
A
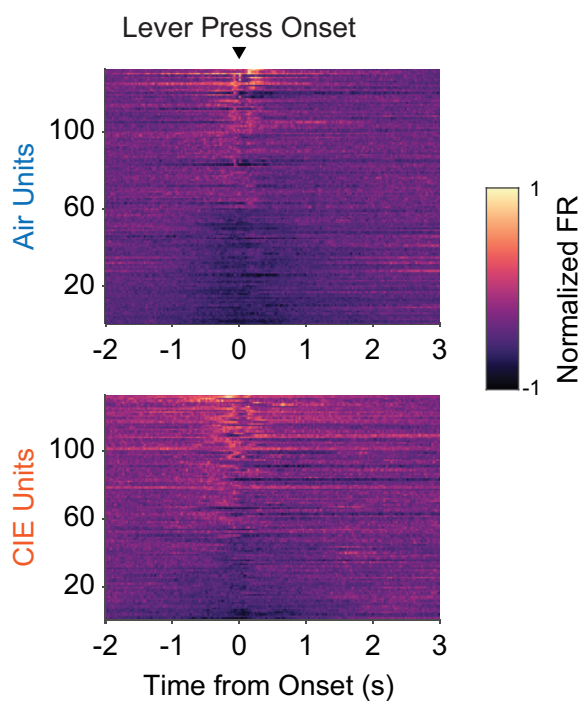

B
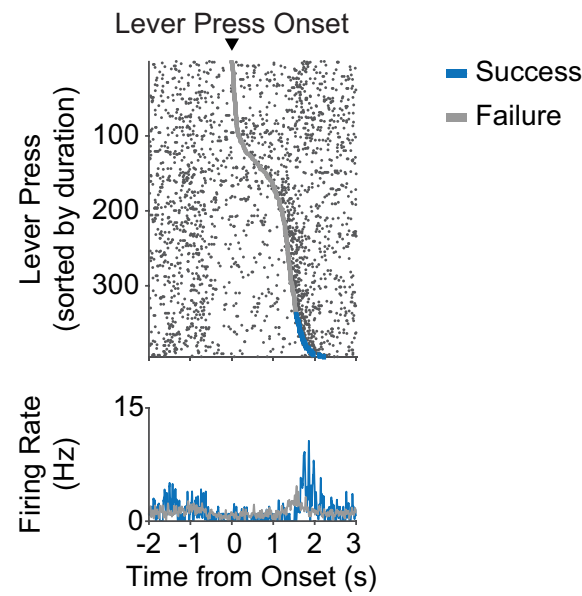

C

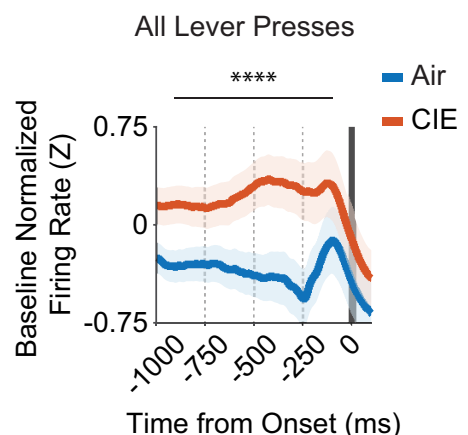

D

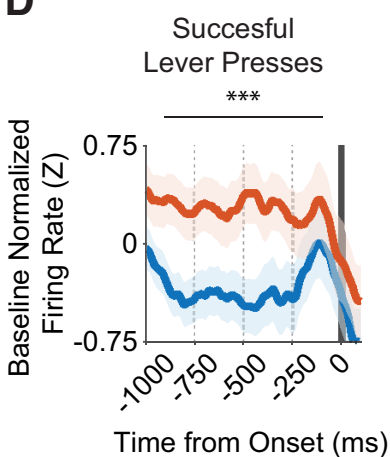

E

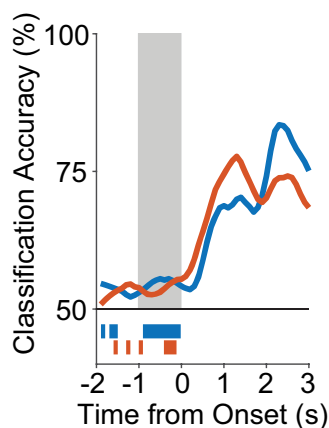

Figure 3. $\boldsymbol{A}-\boldsymbol{E}$, Ethanol dependence alters OFC activity correlates of lever pressing initiation. $\boldsymbol{A}$, Heat map of normalized firing rates for units that significantly increased or decreased from baseline, displayed relative to lever press onset. Units are sorted by activity from a 1-s window around lever press onset. $\boldsymbol{B}$, Raster plot of a representative unit's firing rate relative to lever press onset, sorted from shortest to longest durations within a 1600-ms lever press criterion session that occurred late in training. Gray and blue markers indicate the end of lever presses that failed or succeeded in exceeding the 1600-ms lever press criterion, respectively. C, Average z-scored firing rate changes from baseline for all lever presses and $(\boldsymbol{D})$ successful lever presses only. Firing rate changes were compared across four 250 -ms bins relative to lever press onset. $\boldsymbol{E}$, SVM classification accuracy of task performance outcomes (i.e., did lever press exceed 1600-ms hold-down criterion?) from all captured air and CIE units, displayed relative to lever press onset. Bars underneath traces indicate time points before the onset of the lever press in which classification accuracy was significantly different compared with the null distribution. Shaded region indicates time points in which classification accuracy comparisons were made between air and CIE groups. OFC activity correlates of lever pressing initiation are shown by significantly up-modulated and down-modulated unit averages in Extended Data Figure 3-1. Data points represent mean \pm SEM; ${ }^{\star \star *} p<0.001,{ }^{\star \star \star \star} p<0.0001$

In addition, when we examined only lever presses that were successful, the increased firing rate observed in CIE mice was still present (two-way repeated measures ANOVA (bin $\times$ treatment): main effect of treatment only $\left(F_{(1,1032)}=14.58, p=0.0001\right.$; Fig. $\left.3 D\right)$. We then trained a support vector machine (SVN) model with the peri-event firing rate activity of all captured (including significant and non-significant activity modulation in relation to behavioral epochs) units to directly test whether firing rates could accurately classify whether an individual lever press exceeded the 1600-ms duration criterion. In line with the lack of predictive coding, we found overall low classification performance that did not differ between air and CIE mice. A two-way repeated measures ANOVA (bin $\times$ treatment) comparisons of temporally binned classification performance between air and CIE mice revealed no significant differences within the 1000-ms period preceding lever press onset $(p>0.29)$. Thus, prior CIE procedures increased OFC activity associated with action onset; however, this activity, as well as activity in air control mice, was not predictive of impending lever-press success.

\section{Prior CIE procedures have little effect on OFC activity during lever press execution}

We next asked whether the increased OFC activity observed in CIE mice before lever-press onset would persist as mice held down the lever. Our analysis focused solely on units that were significantly modulated before the onset of a lever press. When we examined individual unit 

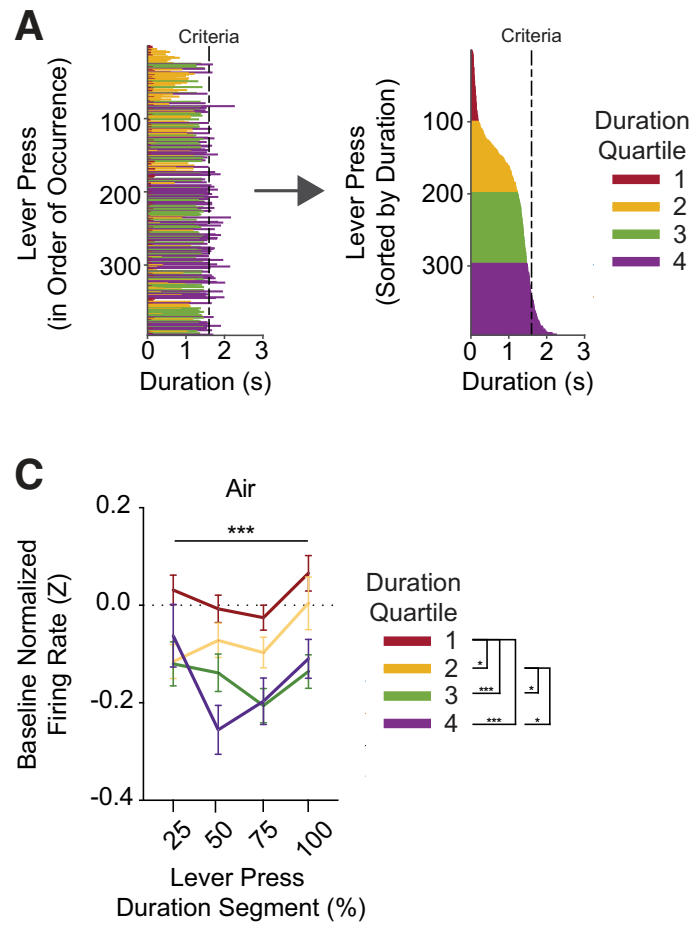
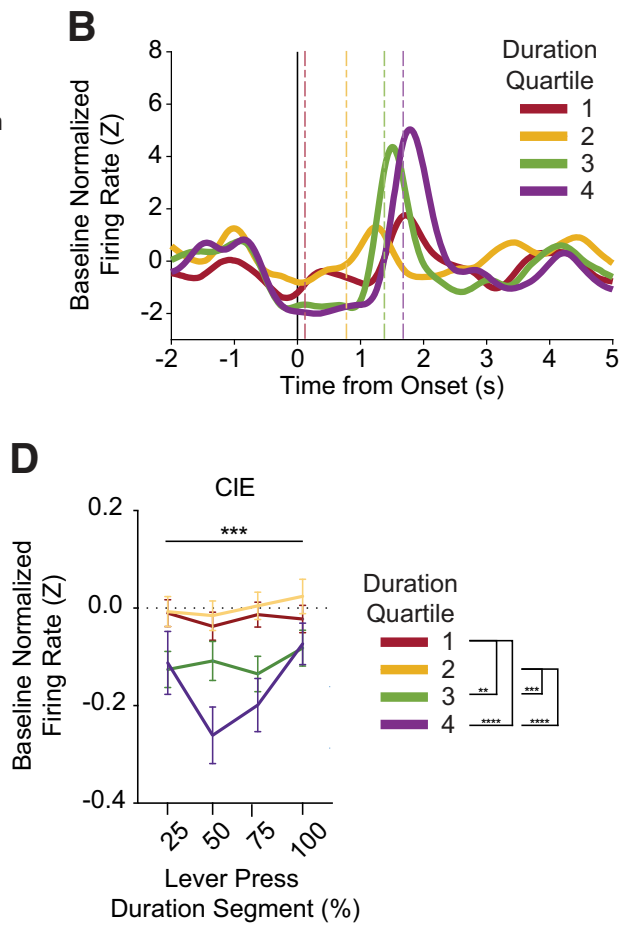

Figure 4. $\boldsymbol{A}-\boldsymbol{D}$, OFC reduces firing while mice hold down the lever. $\boldsymbol{A}$, Lever presses were segmented into four quartiles determined by the distribution of lever press durations within each individual session. $\boldsymbol{B}$, Representative unit's mean baseline normalized zscored firing rate changes displayed relative to lever press onset. Dashed lines indicate the mean lever press duration for each respective duration quartile within the session. $\boldsymbol{C}$, Mean baseline normalized $\mathbf{z}$-scored firing rate changes across equidistant duration segments (i.e., 0-25\%, 25-50\%, 50-75\%, 75-100\% of lever press duration) of lever presses belonging to each duration quartile for air and $(\boldsymbol{D})$ CIE groups. Quartile boundaries determined by within-session lever press duration distributions are shown in Extended Data Figure 4-1. Data points represent mean \pm SEM; ${ }^{*} p<0.05,{ }^{* *} p<0.01,{ }^{* \star *} p<0.001,{ }^{* \star *} p<0.0001$.

activity raster plots (Fig. 3B), we often observed broad reductions in OFC activity as mice held down the lever. Indeed, we found on average only $\sim 38 \%$ of total lever presses made had at least one putative action potential occur during lever press execution, and this was not different in CIE mice (air mice $=37.85 \pm 2 \%$; $\mathrm{CIE}$ mice $=$ $38.25 \pm 2 \%$; unpaired $t$ test with Welch's correction: $\left.t_{(257.4)}=0.15, p=0.88\right)$.

As there was a distribution of lever press durations in each session, we next examined whether this relative reduction in OFC activity was different depending on the duration of the lever press being executed. We also examined whether prior CIE procedures would alter any potential change in activity during the lever press itself. To examine this, for a given mouse on a given day, we first divided lever presses within a session into four quartiles (see Materials and Methods; Fig. 4A). Mean quartile distribution boundaries were similar between groups (Extended Data Fig. $4-1 A, C)$. Then, for each of the lever presses made in those quartiles, spike activity that happened while the mouse was holding down the lever (duration of the press) was z-scored normalized to baseline and divided into four equal segments spanning the lever press duration. This segment $z$-scored activity was then averaged within air and CIE groups. As shown by baseline normalized $z$-scored activity from a representative unit in Figure $4 B$, this allowed us to examine activity changes across the duration of the lever press based on the relative length of the final lever press duration.
We found OFC firing rates during the execution of the lever press did differ depending on the duration of the lever press; however, prior CIE exposure had very little effect on these patterns. As exemplified by Figure $4 B$, Ionger lever presses showed lower firing rates during the lever press, with an increase in firing rate occurring close to the release of the lever press. In air mice, a two-way repeated measures ANOVA (quartile $\times$ segment) revealed a main effect of quartile $\left(F_{(3,2176)}=15.87, p<0.0001\right)$, a main effect of segment $\left(F_{(3,2176)}=4.18, p<0.005\right)$, but no interaction. Follow-up main effect analyses on quartile effects showed that baseline normalized averaged z-scored activity within a quartile largely differed from activity in other quartiles. The exception being activity in the longest two quartiles which did not differ from each other (Fig. 4C). The same analyses in CIE mice showed similar differences (main effect of quartile: $\left.F_{(3,1952)}=15.19, p<0.0001\right)$, but no effect of segment or interaction $(p>0.05)$. In addition, air mice also had similar baseline activity during the first and second (i.e., the shortest lever presses) quartiles (Fig. 4D). Follow-up analyses on main effects of segments showed few differences, except in air mice where the last quarter of the lever press differed from the preceding quarter $(p=0.01)$, as represented by the increase in firing rate during the last portion of a lever press in Figure 4B. Together, the above data suggest that OFC overall reduces its firing rate activity during the execution of the lever press in a manner that reflects the future duration, with longer lever presses showing greater 
A
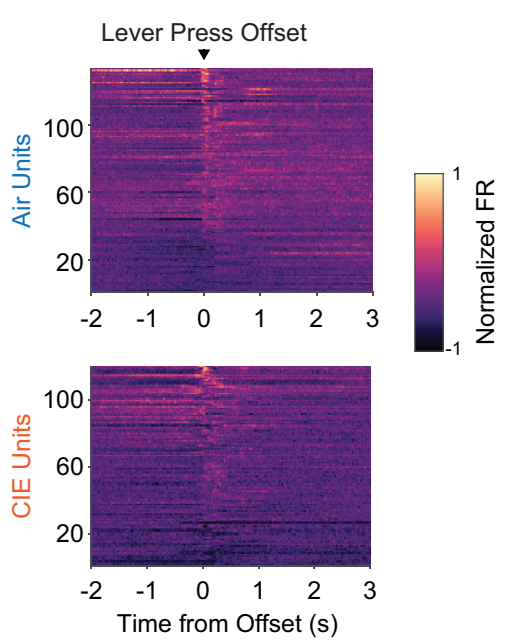

B
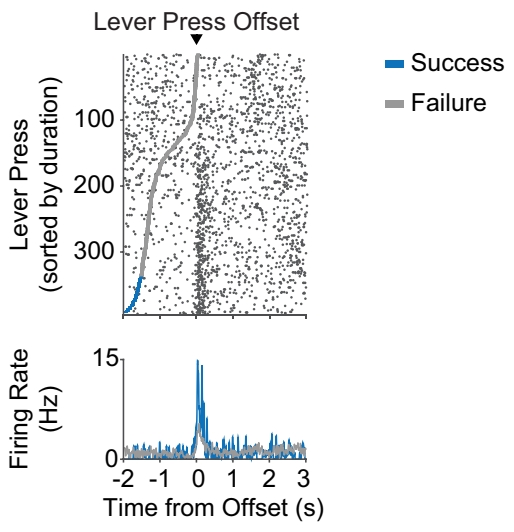

C

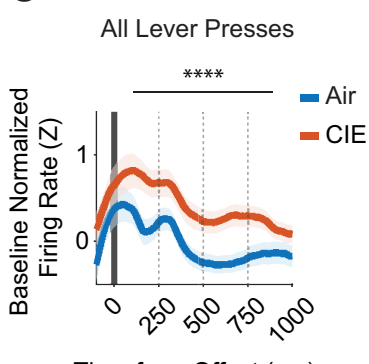

D

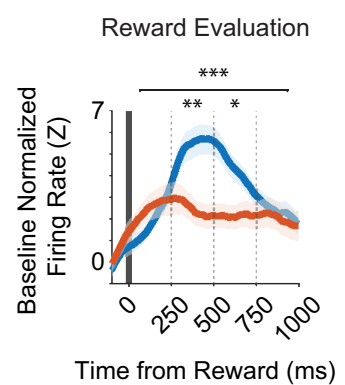

E

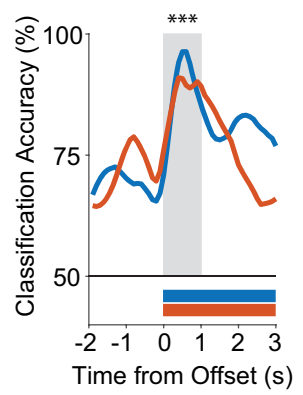

Figure 5. $\boldsymbol{A}-\boldsymbol{E}$, Ethanol dependence alters OFC activity correlates of outcome delivery. $\boldsymbol{A}$, Heat map of normalized firing rates for units that significantly increased or decreased from baseline, displayed relative to lever press offset. Units are sorted by activity from a 1-s window around lever press offset. $\boldsymbol{B}$, Raster plot of a representative unit's firing rate relative to lever press offset, sorted from shortest to longest durations within a 1600-ms lever press criterion session that occurred late in training. Gray and blue markers indicate the start of a lever presses that failed or succeeded in exceeding the 1600-ms lever press criterion, respectively. $\boldsymbol{C}$, Average z-scored firing rate changes from baseline for all lever presses and $(\boldsymbol{D})$ rewarded lever presses only. Firing rate changes were compared across four 250-ms bins relative to lever press offset. $\boldsymbol{E}$, SVM classification accuracy of task performance outcomes (i.e., did lever press exceed 1600-ms hold-down criterion?) from all captured air and CIE units, displayed relative to lever press offset. Bars underneath traces indicate time points after the offset of the lever press in which classification accuracy was significantly different compared with the null distribution. Shaded region indicates time points in which classification accuracy comparisons were made between air and CIE groups. OFC activity correlates of lever pressing termination and reward delivery are shown by significantly up-modulated and down-modulated unit averages in Extended Data Figure 5-1. Data points represent mean \pm SEM; ${ }^{\star} p<0.05,{ }^{* \star} p<0.01,{ }^{* \star *} p<0.001$.

reductions in firing rate, and that CIE does not drastically alter the ability of OFC to do so.

\section{Prior CIE procedures decrease OFC activity during outcomes}

OFC neurons have long been reported to increase their activity in anticipation of and during outcome delivery (Wallis, 2007; Stalnaker et al., 2014, 2018). In the present task, reward delivery cannot occur until the lever is released. Thus, we defined an action offset epoch (1000 $\mathrm{ms}$ ), and in some cases an outcome-related epoch (3000 $\mathrm{ms}$ ) following a reward delivery, that encompassed moving to the food receptacle and potentially reward consumption. As the reward is readily visible without mice having to insert their heads into the food receptacle, it is likely that reward perception happens earlier than consumption. As seen in the normalized activity perievent heatmaps (Fig. $5 A$ ) and in an example from a representative OFC unit (Fig. 5B), OFC firing rates changed significantly during the action offset and outcome-related epochs of the task. We observed similar percentages of OFC units recorded between air and CIE mice where significant activity changes were associated with action offset only (air $=13.5 \%, \mathrm{CIE}=16.5 \%$ ) and outcome-related only (air $=11.5 \%, \mathrm{CIE}=7.5 \%$ ), as well as OFC units that had activity associated with both action offset and outcome evaluation (air 12\%, CIE 7\%) or action onset, action offset, and outcome-related (air = 18\%; CIE = 23\%; Fig. 2E).

When we examined modulation of OFC activity following lever press offset, we found that CIE mice showed greater increases in baseline normalized z-scored OFC firing rates compared with air controls (two-way repeated 
measures ANOVA (bin $\times$ treatment); no interaction; main effect of bin: $F_{(3,1044)}=6.612, p=0.0002$; main effect of treatment: $F_{(1,1044)}=22.63, p<0.0001$; Fig. $5 C$; Extended Data Fig. 5-1A,B). We also found that OFC activity changes at lever press offset reflected performance outcomes in both air and CIE mice (Extended Data Fig. 5-1E, $F$ ); however, it is important to note that this OFC activity was comprised of all lever presses, including those that were rewarded. Thus, we examined OFC firing rate changes aligned to outcome-related epochs following only successful lever presses and asked whether CIE procedures would change the magnitude of these increases. We found large increases in OFC firing rate changes during outcome-related epochs. In contrast to the increase in activity related to lever-pressing in CIE mice, we found air mice had greater increases in OFC firing rates during outcome-related epochs compared with CIE mice (two-way repeated measures ANOVA (bin $\times$ treatment); interaction: $F_{(3,724)}=4.04, p=0.007$; main effect of bin: $F_{(3,724)}=4.43$, $p=0.004$; main effect of treatment: $F_{(1,724)}=8.66$, $p=0.003$; Fig. 5D; Extended Data Fig. 5-1C,D). Given the overall robust increases in OFC activity during outcomerelated epochs, as well as the differences in magnitude induced by CIE procedures, we asked whether a support vector machine (SVM) model trained with the peri-event activity of all captured OFC units could accurately classify whether an individual lever press exceeded the 1600-ms duration criterion during the action offset and outcomerelated epochs. We found high classification accuracy during the outcome-related epochs, especially within the first $1000 \mathrm{~ms}$ of reward delivery. Furthermore, classification accuracy was lower in OFC units from CIE mice during this period (two-way repeated measures ANOVA (bin $\times$ treatment); interaction: $\left(F_{(9,180)}=3.33, p=0.0009\right.$; main effect of bin: $\left.F_{(9,180)}=5.11, p<0.0001\right)$. A post hoc Benjamini and Hochberg multiple comparison test revealed that decoder accuracy differences were pronounced in the 1st $(p=0.005)$, 3rd $(p=0.006)$, and 4th $(p=0.0086) 100-\mathrm{ms}$ bins of the outcome related epoch. Thus, CIE mice show greater OFC activity associated with lever pressing, but reduced OFC activity during outcomerelated epochs, with OFC activity being less predictive of rewarded lever presses.

\section{Discussion}

Alcohol dependence is associated with impairments to OFC function and aberrant decision-making, thereby increasing the vulnerability to relapse and maladaptive alcohol consumption (Zinn et al., 2004; Chanraud et al., 2007; Loeber et al., 2009; Berre et al., 2012; Reiter et al., 2016; Le Berre et al., 2017). Here, we uncovered neural correlates of actions and outcomes and found them perturbed by prior chronic alcohol exposure and withdrawal. Our results suggest alcohol exposure induces long-lasting perturbations to OFC activity in a bidirectional manner, dependent on the computation being performed. CIE mice showed a modulation of OFC activity that suggests overall increases in OFC activity associated with actions (i.e., lever press onset and offset), and a blunting of OFC activity during outcome-related epochs. This raises the hypothesis that alcohol dependence does not result in a loss of OFC recruitment, but rather induces a change in how computations performed by OFC circuits may contribute to decision-making.

In the present data, CIE-exposed animals acquired and performed the lever pressing at similar levels compared with alcohol naive controls (Fig. 1). However, outcome devaluation testing showed that such lever pressing was not under goal-directed control as it was in air mice. Thus, $\mathrm{CIE}$ mice were able to acquire lever pressing for food relying on neural mechanisms supporting habit learning. Recent findings corroborate the observed lack of goal-directed control in CIE mice (Lopez et al., 2014; Renteria et al., 2018, 2020; Barker et al., 2020) and with the disruption to decision-making control observed under different instrumental tasks and varied tests of goal-directed control. Our work adds to an ever-growing body of research on such decision-making deficits and highlights the importance of examining alcohol-induced alterations to neural circuits and mechanisms controlling goal-directed processes.

Clinical studies have previously shown that alcohol dependence alters representation of decision-making within OFC circuits, albeit not always in the same manner. The OFC is widely found to be hypoactive in alcohol dependence (Volkow et al., 1994, 1997; Boettiger et al., 2007; Sjoerds et al., 2013; Reiter et al., 2016), but there have also been reports of hyperactivity (Wrase et al., 2002; Tapert et al., 2003; Myrick et al., 2004, 2008; Hermann et al., 2006; Ernst et al., 2014; Reinhard et al., 2015). We find that to be the case in our data as well, such that alcohol exposure altered decision-making representations in the OFC in a variety of ways. Prior CIE exposure and withdrawal changed OFC computations in a way that suggests an overall increase in activity during actions (Fig. 3). This dependence-induced change could suggest an increased contribution of OFC processes to action-related processes. We should emphasize that the action contingency in the present task is the duration of the lever press, and that it is inferred from prior experience. This suggests that alcohol dependence increases OFC activity related to the retrieval and execution of inferred action association. In this context, it is important to note that a hyperactive OFC has also been observed in those with obsessive compulsive disorder and increased activity of OFC neurons during actions has been linked to compulsive action phenotypes (Milad and Rauch, 2012; Pauls et al., 2014; Robbins et al., 2019; Lüscher et al., 2020). Whether this increased OFC activity related to actions that we find in CIE mice plays a role in compulsive phenotypes in alcohol dependence is not currently known.

A hallmark of OFC function is its contribution to reward evaluation and updating, with increases in OFC activity observed during outcome anticipation and presentation (Rolls et al., 1996; Wallis, 2011; Jones et al., 2012; Stalnaker et al., 2014, 2015, 2018). Recent works have shown that inhibition of OFC activity during periods of outcome presentation prevent outcome evaluation and updating (Baltz et al., 2018; Malvaez et al., 2019). Further, recent work in humans has suggested that OFC encodes 
reward identity expectations (Howard and Kahnt, 2018), which contribute to the generation of prediction errors even when there is no change in value (Stalnaker et al., 2018). Here, we observed large increases in OFC activity during outcome-evaluation periods and this increase was reduced following alcohol exposure (Fig. 5). SVM modeling showed reduced accuracy in classifying rewarded lever presses following alcohol exposure. Furthermore, $\mathrm{CIE}$ affected OFC computations made only after successful lever presses. Thus, in addition to action-associated OFC activity, the data above strongly suggests that OFC's contribution to outcome retrieval, evaluation, or identification is altered following alcohol exposure. Further, results of from decoder analysis showed that OFC activity during outcome-related epochs normally carries information about whether the lever press was successful or not. CIE reduced this OFC representation. Combined with the insensitivity to outcome devaluation, our data support the hypothesis that alcohol dependence leads to reduced contribution of OFC to outcome-related decision-making. Further, the observed bidirectional alcohol exposure effects on OFC computations support the hypothesized complexity of alcohol dependence effects on OFC decision-making circuitry. For instance, previous accounts on the modulatory influence of alcohol dependence on OFC activity have differed (Volkow et al., 1994, 1997; Boettiger et al., 2007; Sjoerds et al., 2013; Reiter et al., 2016), which in conjunction with our findings suggests a divergent effect of alcohol dependence that may be dependent on decision-making demands and information in OFC. While the critical role for the OFC in regulating the ability to adapt behavior when outcome value or identity changes has been largely established, here we present new evidence on the specificity of dependence-induced effects on the computations supporting these processes.

The self-paced nature of our task allowed us to investigate the dynamics of OFC computations made during ongoing decision-making that relies solely on internal representations or retrieval of learned duration contingencies, rather than a reliance on predictive external sensory information. Here, we show with in vivo electrophysiology data that the OFC activity was modulated during the lever press itself. OFC activity decreased while mice held down the lever, with OFC activity in air controls resembling a U-shaped pattern during longer presses (Fig. 4). The continuous nature of holding down the lever revealed an activity pattern of an initial decrease in activity relative to baseline, that subsequently increases before the release of the lever press and before when outcomes are expected. The increase in activity before lever press release may correspond to a greater confidence in outcome delivery, something previously shown for OFC activity in cued tasks (Kepecs et al., 2008; Masset et al., 2020). Another possibility stems from prior data from OFC lesioned patients suggesting a potential role for OFC activity in evaluating the passing of time (Berlin et al., 2004; Berlin and Rolls, 2004). Considering reports of increased OFC activity during actions and its association with compulsive action phenotypes similar to those seen in drug studies (Milad and Rauch, 2012; Pauls et al., 2014; Robbins et al.,
2019; Lüscher et al., 2020), another hypothesis could be that we would observe larger increases in OFC activity at some point during the duration of lever presses and that $\mathrm{CIE}$ may enhance this. Overall, prior alcohol exposure had very minor effects on duration-related activity patterns, although the increase before lever press release was absent in CIE mice. Thus, future experiments aimed at investigating the above hypotheses are warranted.

The dichotomy of CIE effects on the different behavioral components of our task suggests a combination of OFC circuitry changes that could manifest in a variety of ways. In the future, it will be of interest to examine how CIE perturbs OFC function and output that relies on information received by interconnected structures. For example, chronic alcohol exposure and withdrawal may be perturbing the excitability and transmission of local OFC circuitry via cell-type-specific changes (Badanich et al., 2013; McGuier et al., 2015; Nimitvilai et al., 2017; Renteria et al., 2018), such that the integration of incoming information from other associative regions necessary to guide decision-making is disrupted. Additional difficulties in parsing the effects of alcohol dependence on decision-making processes arise from a fundamental lack of structural and functional input-output mapping of the highly complex neural circuits that support decision-making. While in general the areas projecting to OFC have been identified, the relative proportions of inputs across brain regions, as well as the connectivity, strength, and pattern of inputs onto excitatory and inhibitory OFC populations is unknown in naive circumstances, much less following alcohol dependence. It may be that alcohol dependence results in a redistribution of inputs across OFC excitatory and inhibitory populations and/or alters input transmission onto OFC circuits, thereby altering their ability to contribute to decision-making. We should note that it is not clear whether effects observed on neural activity in the present study differ depending on sex. Because of difficulties in female mice maintaining and carrying electrodes and associated head-caps, we were not powered in our in vivo recording experiments to examine whether there were any sex differences in our neural data that could differentially mediate contingency and expected outcome value control (Barker et al., 2010).

As with all brain areas, the capacity to contribute to decision-making computations is going to depend on the afferent inputs as well as local capabilities. Alcohol dependence is likely to affect both across the brain. Here, we identified some of the complexity in how OFC's contributions to decision-making computations are altered following alcohol exposure. These findings will hopefully shed light on the behavioral and OFC-based perturbations previously reported and provide insight into the therapeutic treatment of alcohol dependence.

\section{References}

Ahmari SE, Spellman T, Douglass NL, Kheirbek MA, Simpson HB, Deisseroth K, Gordon JA, Hen R (2013) Repeated cortico-striatal stimulation generates persistent OCD-like behavior. Science 340:1234-1239.

Badanich KA, Becker HC, Woodward JJ (2011) Effects of chronic intermittent ethanol exposure on orbitofrontal and medial prefrontal 
cortex-dependent behaviors in mice. Behav Neurosci 125:879891.

Badanich KA, Mulholland PJ, Beckley JT, Trantham-Davidson H, Woodward JJ (2013) Ethanol reduces neuronal excitability of lateral orbitofrontal cortex neurons via a glycine receptor dependent mechanism. Neuropsychopharmacology 38:1176-1188.

Baltz ET, Yalcinbas EA, Renteria R, Gremel CM (2018) Orbital frontal cortex updates state-induced value change for decision-making. ELife 7:e35988.

Barker JM, Torregrossa MM, Arnold AP, Taylor JR (2010) Dissociation of genetic and hormonal influences on sex differences in alcoholism-related behaviors. J Neurosci 30:9140-9144.

Barker JM, Bryant KG, Montiel-Ramos A, Goldwasser B, Chandler LJ (2020) Selective deficits in contingency-driven ethanol seeking following chronic ethanol exposure in male mice. Alcohol Clin Exp Res 44:1896-1905.

Beck A, Wüstenberg T, Genauck A, Wrase J, Schlagenhauf F, Smolka MN, Mann K, Heinz A (2012) Effect of brain structure, brain function, and brain connectivity on relapse in alcohol-dependent patients. Arch Gen Psychiatry 69:842-852.

Becker HC, Hale RL (1993) Repeated episodes of ethanol withdrawal potentiate the severity of subsequent withdrawal seizures: an animal model of alcohol withdrawal "kindling". Alcohol Clin Exp Res 17:94-98.

Becker HC, Lopez MF (2004) Increased ethanol drinking after repeated chronic ethanol exposure and withdrawal experience in C57BL/6 mice. Alcohol Clin Exp Res 28:1829-1838.

Berlin HA, Rolls ET (2004) Time perception, impulsivity, emotionality, and personality in self-harming borderline personality disorder patients. J Pers Disord 18:358-378.

Berlin HA, Rolls ET, Kischka U (2004) Impulsivity, time perception, emotion and reinforcement sensitivity in patients with orbitofrontal cortex lesions. Brain 127:1108-1126.

Berre APL, Vabret F, Cauvin C, Pinon K, Allain P, Pitel AL, Eustache $F$, Beaunieux $H$ (2012) Cognitive barriers to readiness to change in alcohol-dependent patients. Alcohol Clin Exp Res 36:1542-1549.

Bickel WK, Mellis AM, Snider SE, Athamneh LN, Stein JS, Pope DA (2018) 21st century neurobehavioral theories of decision making in addiction: review and evaluation. Pharmacol Biochem Behav 164:4-21.

Boettiger CA, Mitchell JM, Tavares VC, Robertson M, Joslyn G, D'Esposito M, Fields HL (2007) Immediate reward bias in humans: fronto-parietal networks and a role for the catechol-O-methyltransferase 158Val/Val genotype. J Neurosci 27:14383-14391.

Burguière E, Monteiro P, Feng G, Graybiel AM (2013) Optogenetic stimulation of lateral orbitofronto-striatal pathway suppresses compulsive behaviors. Science 340:1243-1246.

Cardenas VA, Durazzo TC, Gazdzinski S, Mon A, Studholme C, Meyerhoff DJ (2011) Brain morphology at entry into treatment for alcohol dependence is related to relapse propensity. Biol Psychiatry 70:561-567.

Chanraud S, Martelli C, Delain F, Kostogianni N, Douaud G, Aubin HJ, Reynaud M, Martinot JL (2007) Brain morphometry and cognitive performance in detoxified alcohol-dependents with preserved psychosocial functioning. Neuropsychopharmacology 32:429438.

Corbit LH, Nie H, Janak PH (2012) Habitual alcohol seeking: time course and the contribution of subregions of the dorsal striatum. Biol Psychiatry 72:389-395.

Dias-Ferreira E, Sousa JC, Melo I, Morgado P, Mesquita AR, Cerqueira JJ, Costa RM, Sousa N (2009) Chronic stress causes frontostriatal reorganization and affects decision-making. Science 325:621-625.

Dickinson A (1985) Actions and habits: the development of behavioural autonomy. Philos Trans R Soc Lond B Biol Sci 308:67.

Durazzo TC, Tosun D, Buckley S, Gazdzinski S, Mon A, Fryer SL, Meyerhoff DJ (2011) Cortical thickness, surface area, and volume of the brain reward system in alcohol dependence: relationships to relapse and extended abstinence. Alcohol Clin Exp Res 35:11871200.
Ernst LH, Plichta MM, Dresler T, Zesewitz AK, Tupak SV, Haeussinger FB, Fischer M, Polak T, Fallgatter AJ, Ehlis A-C (2014) Prefrontal correlates of approach preferences for alcohol stimuli in alcohol dependence. Addict Biol 19:497-508.

Fan D, Rossi MA, Yin HH (2012) Mechanisms of action selection and timing in substantia nigra neurons. J Neurosci 32:5534-5548.

Fellows LK (2007) The role of orbitofrontal cortex in decision making: a component process account. Ann NY Acad Sci 1121:421-430.

Fernandez GM, Lew BJ, Vedder LC, Savage LM (2017) Chronic intermittent ethanol exposure leads to alterations in brain-derived neurotrophic factor within the frontal cortex and impaired behavioral flexibility in both adolescent and adult rats. Neuroscience 348:324-334.

Gourley SL, Olevska A, Zimmermann KS, Ressler KJ, DiLeone RJ, Taylor JR (2013) The orbitofrontal cortex regulates outcomebased decision-making via the lateral striatum. Eur $\mathrm{J}$ Neurosci 38:2382-2388.

Gremel CM, Costa RM (2013) Orbitofrontal and striatal circuits dynamically encode the shift between goal-directed and habitual actions. Nat Commun 4:2264.

Gremel CM, Chancey JH, Atwood BK, Luo G, Neve R, Ramakrishnan C, Deisseroth K, Lovinger DM, Costa RM (2016) Endocannabinoid modulation of orbitostriatal circuits gates habit formation. Neuron 90:1312-1324.

Griffin WC, Lopez MF, Becker HC (2009) Intensity and duration of chronic ethanol exposure is critical for subsequent escalation of voluntary ethanol drinking in mice. Alcohol Clin Exp Res 33:18931900.

Heilig M, Egli M, Crabbe JC, Becker HC (2010) Acute withdrawal, protracted abstinence and negative affect in alcoholism: are they linked? Addict Biol 15:169-184.

Hermann D, Smolka MN, Wrase J, Klein S, Nikitopoulos J, Georgi A, Braus DF, Flor H, Mann K, Heinz A (2006) Blockade of cue-induced brain activation of abstinent alcoholics by a single administration of amisulpride as measured with fMRI. Alcohol Clin Exp Res 30:1349-1354.

Howard JD, Kahnt T (2018) Identity prediction errors in the human midbrain update reward-identity expectations in the orbitofrontal cortex. Nat Commun 9:1611.

Jin X, Costa RM (2010) Start/stop signals emerge in nigrostriatal circuits during sequence learning. Nature 466:457-462.

Jones JL, Esber GR, McDannald MA, Gruber AJ, Hernandez A, Mirenzi A, Schoenbaum G (2012) Orbitofrontal cortex supports behavior and learning using inferred but not cached values. Science 338:953-956.

Kepecs A, Uchida N, Zariwala HA, Mainen ZF (2008) Neural correlates, computation and behavioural impact of decision confidence. Nature 455:227-231.

Kroener S, Mulholland PJ, New NN, Gass JT, Becker HC, Chandler LJ (2012) Chronic alcohol exposure alters behavioral and synaptic plasticity of the rodent prefrontal cortex. PLoS One 7:e37541.

Kuch DO (1974) Differentiation of press durations with upper and lower limits on reinforced values. J Exp Anal Behav 22:275-283.

Laakso MP, Gunning-Dixon F, Vaurio O, Repo-Tiihonen E, Soininen $\mathrm{H}$, Tiihonen $\mathrm{J}$ (2002) Prefrontal volumes in habitually violent subjects with antisocial personality disorder and type 2 alcoholism. Psychiatry Res 114:95-102.

Le Berre AP, Fama R, Sullivan EV (2017) Executive functions, memory, and social cognitive deficits and recovery in chronic alcoholism: a critical review to inform future research. Alcohol Clin Exp Res 41:1432-1443.

Loeber S, Duka T, Welzel H, Nakovics H, Heinz A, Flor H, Mann K (2009) Impairment of cognitive abilities and decision making after chronic use of alcohol: the impact of multiple detoxifications. Alcohol Alcohol 44:372-381.

Lopez MF, Becker HC (2005) Effect of pattern and number of chronic ethanol exposures on subsequent voluntary ethanol intake in C57BL/6J mice. Psychopharmacology (Berl) 181:688-696. 
Lopez MF, Becker HC, Chandler LJ (2014) Repeated episodes of chronic intermittent ethanol promote insensitivity to devaluation of the reinforcing effect of ethanol. Alcohol 48:639-645.

Lüscher C, Robbins TW, Everitt BJ (2020) The transition to compulsion in addiction. Nat Rev Neurosci 21:247-263.

Malvaez M, Shieh C, Murphy MD, Greenfield VY, Wassum KM (2019) Distinct cortical-amygdala projections drive reward value encoding and retrieval. Nat Neurosci 22:762-769.

Masset P, Ott T, Lak A, Hirokawa J, Kepecs A (2020) Behavior- and modality-general representation of confidence in orbitofrontal cortex. Cell 182:112-126.e18.

McGuier NS, Padula AE, Lopez MF, Woodward JJ, Mulholland PJ (2015) Withdrawal from chronic intermittent alcohol exposure increases dendritic spine density in the lateral orbitofrontal cortex of mice. Alcohol 49:21-27.

Meyers EM (2013) The neural decoding toolbox. Front Neuroinform 7:8.

Milad MR, Rauch SL (2012) Obsessive-compulsive disorder: beyond segregated cortico-striatal pathways. Trends Cogn Sci 16:43-51.

Morisot N, Phamluong K, Ehinger Y, Berger AL, Moffat JJ, Ron D (2019) MTORC1 in the orbitofrontal cortex promotes habitual alcohol seeking. ELife 8:e51333.

Myrick H, Anton RF, Li X, Henderson S, Drobes D, Voronin K, George MS (2004) Differential brain activity in alcoholics and social drinkers to alcohol cues: relationship to craving. Neuropsychopharmacology 29:393-402.

Myrick H, Anton RF, Li X, Henderson S, Randall PK, Voronin K (2008) Effect of naltrexone and ondansetron on alcohol cue-induced activation of the ventral striatum in alcohol-dependent people. Arch Gen Psychiatry 65:466-475.

Nimitvilai S, Lopez MF, Mulholland PJ, Woodward JJ (2016) Chronic Intermittent ethanol exposure enhances the excitability and synaptic plasticity of lateral orbitofrontal cortex neurons and induces a tolerance to the acute inhibitory actions of ethanol. Neuropsychopharmacology 41:1112-1127.

Nimitvilai S, Uys JD, Woodward JJ, Randall PK, Ball LE, Williams RW, Jones BC, Lu L, Grant KA, Mulholland PJ (2017) Orbitofrontal neuroadaptations and cross-species synaptic biomarkers in heavy-drinking macaques. J Neurosci 37:3646-3660.

Padoa-Schioppa C, Conen KE (2017) Orbitofrontal cortex: a neural circuit for economic decisions. Neuron 96:736-754.

Pascoli V, Terrier J, Hiver A, Lüscher C (2015) Sufficiency of mesolimbic dopamine neuron stimulation for the progression to addiction. Neuron 88:1054-1066.

Pascoli V, Hiver A, Van Zessen R, Loureiro M, Achargui R, Harada M, Flakowski J, Lüscher C (2018) Stochastic synaptic plasticity underlying compulsion in a model of addiction. Nature 564:366-371.

Pauls DL, Abramovitch A, Rauch SL, Geller DA (2014) Obsessivecompulsive disorder: an integrative genetic and neurobiological perspective. Nat Rev Neurosci 15:410-424.

Pereira EF, Aracava Y, Aronstam RS, Barreiro EJ, Albuquerque EX (1992) Pyrazole, an alcohol dehydrogenase inhibitor, has dual effects on N-methyl-D-aspartate receptors of hippocampal pyramidal cells: agonist and noncompetitive antagonist. J Pharmacol Exp Ther 261:331-340.

Platt JR, Kuch DO, Bitgood SC (1973) Rats' lever-press durations as psychophysical judgements of time. J Exp Anal Behav 19:239-250.

Reich RR, Goldman MS (2015) Decision making about alcohol use: the case for scientific convergence. Addict Behav 44:23-28.

Reinhard I, Leménager T, Fauth-Bühler M, Hermann D, Hoffmann S, Heinz A, Kiefer F, Smolka MN, Wellek S, Mann K, Vollstädt-Klein S (2015) A comparison of region-of-interest measures for extracting whole brain data using survival analysis in alcoholism as an example. J Neurosci Methods 242:58-64.

Reiter AMF, Deserno L, Kallert T, Heinze H-J, Heinz A, Schlagenhauf $F$ (2016) Behavioral and neural signatures of reduced updating of alternative options in alcohol-dependent patients during flexible decision-making. J Neurosci 36:10935-10948.
Renteria R, Baltz ET, Gremel CM (2018) Chronic alcohol exposure disrupts top-down control over basal ganglia action selection to produce habits. Nat Commun 9:211.

Renteria R, Cazares C, Gremel CM (2020) Habitual ethanol seeking and licking microstructure of enhanced ethanol self-administration in ethanol-dependent mice. Alcohol Clin Exp Res 44:880-891.

Rhodes SEV, Murray EA (2013) Differential effects of amygdala, orbital prefrontal cortex, and prelimbic cortex lesions on goal-directed behavior in rhesus macaques. J Neurosci 33:3380-3389.

Robbins TW, Vaghi MM, Banca P (2019) Obsessive-compulsive disorder: puzzles and prospects. Neuron 102:27-47.

Rolls ET, Everitt BJ, Roberts A (1996) The orbitofrontal cortex. Philos Trans R Soc Lond B Biol Sci 351:1433-1444; discussion 14431444.

Sebold M, Nebe S, Garbusow M, Guggenmos M, Schad DJ, Beck A, Kuitunen-Paul S, Sommer C, Frank R, Neu P, Zimmermann US, Rapp MA, Smolka MN, Huys QJM, Schlagenhauf F, Heinz A (2017) When habits are dangerous: alcohol expectancies and habitual decision making predict relapse in alcohol dependence. Biol Psychiatry 82:847-856.

Shoham S, Fellows MR, Normann RA (2003) Robust, automatic spike sorting using mixtures of multivariate t-distributions. J Neurosci Methods 127:111-122.

Siegle JH, López AC, Patel YA, Abramov K, Ohayon S, Voigts J (2017) OpenEphys: an open-source, plugin-based platform for multichannel electrophysiology. J Neural Eng 14:e045003.

Sjoerds Z, de Wit S, van den Brink W, Robbins TW, Beekman ATF, Penninx BWJH, Veltman DJ (2013) Behavioral and neuroimaging evidence for overreliance on habit learning in alcohol-dependent patients. Transl Psychiatry 3:e337.

Stalnaker TA, Cooch NK, McDannald MA, Liu TL, Wied H, Schoenbaum G (2014) Orbitofrontal neurons infer the value and identity of predicted outcomes. Nat Commun 5:3926.

Stalnaker TA, Cooch NK, Schoenbaum G (2015) What the orbitofrontal cortex does not do. Nat Neurosci 18:620-627.

Stalnaker TA, Liu T-L, Takahashi YK, Schoenbaum G (2018) Orbitofrontal neurons signal reward predictions, not reward prediction errors. Neurobiol Learn Mem 153:137-143.

Stephens DN, Duka T (2008) Cognitive and emotional consequences of binge drinking: role of amygdala and prefrontal cortex. Philos Trans R Soc Lond B Biol Sci 363:3169-3179.

Tapert SF, Cheung EH, Brown GG, Frank LR, Paulus MP, Schweinsburg AD, Meloy MJ, Brown SA (2003) Neural response to alcohol stimuli in adolescents with alcohol use disorder. Arch Gen Psychiatry 60:727-735.

Thayer RE, Hagerty SL, Sabbineni A, Claus ED, Hutchison KE, Weiland BJ (2016) Negative and interactive effects of sex, aging, and alcohol abuse on gray matter morphometry. Hum Brain Mapp 37:2276-2292.

Volkow ND, Wang GJ, Hitzemann R, Fowler JS, Overall JE, Burr G, Wolf AP (1994) Recovery of brain glucose metabolism in detoxified alcoholics. Am J Psychiatry 151:178-183.

Volkow ND, Wang GJ, Overall JE, Hitzemann R, Fowler JS, Pappas N, Frecska E, Piscani K (1997) Regional brain metabolic response to lorazepam in alcoholics during early and late alcohol detoxification. Alcohol Clin Exp Res 21:1278-1284.

Wallis JD (2007) Orbitofrontal cortex and its contribution to decisionmaking. Annu Rev Neurosci 30:31-56.

Wallis JD (2011) Cross-species studies of orbitofrontal cortex and value-based decision-making. Nat Neurosci 15:13-19.

Wrase J, Grüsser SM, Klein S, Diener C, Hermann D, Flor H, Mann K, Braus DF, Heinz A (2002) Development of alcohol-associated cues and cue-induced brain activation in alcoholics. Eur psychiatry 17:287-291.

Yin $\mathrm{HH}$ (2009) The role of the murine motor cortex in action duration and order. Front Integr Neurosci 3:23.

Zinn S, Stein R, Swartzwelder HS (2004) Executive functioning early in abstinence from alcohol. Alcohol Clin Exp Res 28:1338-1346. 